\section{First therapeutic cancer vaccine approved}

The US FDA approved sipuleucel-T (Provenge; Dendreon) on 29 April 2010 for the treatment of men with metastatic castration-resistant prostate cancer.

The lowdown: Sipuleucel-T is the first autologous cellular immunotherapy to be approved by a major regulatory authority. The treatment involves isolating dendritic cells from a patient and treating them with the prostate-specific antigen prostatic acid phosphatase (PAP), which

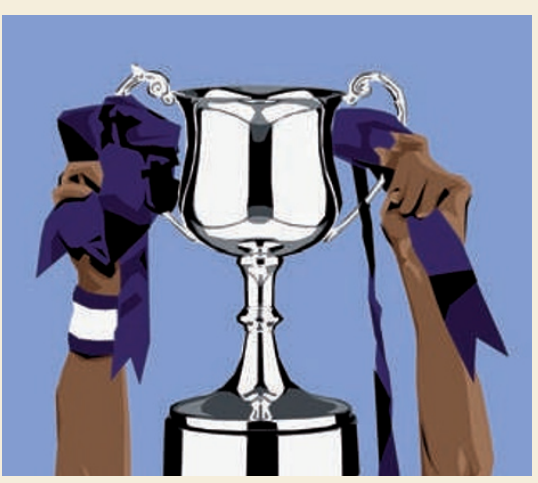
is found in $95 \%$ of prostate cancers. Once returned to the patient, these activated dendritic cells should, in principle, activate T cells to attack and destroy cancer cells that are expressing PAP.

The prostate cancer community has been waiting for the FDA's decision to approve sipuleucel-T since March 2007 (Nature Rev. Drug Discov. 6, 333-334; 2007). At this time, the FDA's Office of Cellular, Tissue and Gene Therapies Advisory Committee voted 17-0 that sipuleucel-T was safe and 13-4 in favour of its efficacy. However, in an approvable letter that the company received in May 2007, the FDA requested more information, including additional clinical efficacy data.

In April 2009, Dendreon announced that it had completed the IMPACT (immunotherapy for prostate adenocarcinoma treatment) trial, and sipuleucel-T met its primary end point by extending median survival by 4.1 months compared with placebo and improving 3-year survival by $38 \%$. The final submission of the biologics license application for sipuleucel-T was completed in November 2009.

The approval of sipuleucel-T provides a major boost to the cancer vaccine field, given that nearly all cancer vaccines evaluated over the past 5 years have not demonstrated clinically meaningful benefits. One of the reasons why researchers think cancer vaccines tested so far often have not shown the same efficacy in humans as in animal studies is because they have been evaluated in cancer patients with advanced stages of disease, who have compromised immune systems. This has led to the suggestion that cancer immunotherapies should be combined with immune-modulatory strategies to counter immune suppression, and also that trial designs need to be adjusted to take into account the differences in the nature of responses to such immunotherapies compared with those for traditional anticancer drugs (Nature Rev. Drug Discov. 8, 685-686; 2009).

\section{FDA rejects orphan drug for lung disease}

Despite receiving a positive advisory committee vote of 9-3, the US FDA rejected InterMune's new drug application for pirfenidone, an orphan drug in development for the treatment of idiopathic pulmonary fibrosis.

The lowdown: Idiopathic pulmonary fibrosis (IPF) - a chronic and life-threatening lung disease characterized by progressive lung scarring that can cause respiratory failure - has a mortality rate that exceeds that of many cancers. In the past, development of new therapies for IPF has been hampered by difficulties in classifying the disease and associated conditions, which has led to misdiagnosis and therapeutic strategies that delivered little clinical benefit (Nature Rev. Drug Discov. 9, 129-140; 2010).

Currently, there is no approved therapy for the treatment of IPF, so the development of pirfenidone - a pyridone compound with unknown mechanism of action that has shown wide-ranging effects including antifibrotic, anti-inflammatory and antioxidant activity in animal models - has attracted considerable interest. Of the two pivotal Phase III clinical trials from which data were submitted as part of InterMune's new drug application for Capacity 2 met the primary end point of a $50 \%$ reduction in the rate of decreased lung function capacity compared with placebo. Nevertheless, when the FDA's Pulmonary-Allergy Drugs Advisory Committee assessed both trials, as part of InterMune's new drug application, the knowledge that only one pivotal trial met pirfenidone, known as Capacity 1 and 2, only its primary end point did not prevent them from voting 9-3 recommending its approval. However, the FDA issued a complete response letter to InterMune requesting an additional clinical trial to support the efficacy of pirfenidone in patients with IPF. Now, InterMune intends to meet with the FDA as soon as possible to discuss pathways to approval. In the meantime, the company remains hopeful that the marketing authorization application that it submitted to the European Medicines Agency on 2 March 2010 will be successful.

\section{European regulator issues pharmacogenetics guidance}

The European Medicines Agency has released a draft guideline on the use of pharmacogenetic methods in the evaluation of the pharmacokinetics of new therapies. The lowdown: Assessing the pharmacokinetics of an investigational drug is an essential part of preclinical and early drug development. Data from these studies are used to predict drug interaction potential, to estimate and understand variability in drug disposition and to select doses for clinical trials.

It is well established that variations such as single nucleotide polymorphisms in genes coding for proteins involved in drug metabolism, such as cytochrome P450 enzymes, can have an important influence on drug pharmacokinetics (Nature Rev. Drug Discov. 7, 293-305; 2008). For example, the product label for the anticoagulant warfarin was updated in 2007 to note that patients with certain variants of two genes involved in drug metabolism, CYP2C9 and VKORC1, require lower doses of warfarin than average to obtain the same therapeutic effect, and are more likely to suffer from bleeding complications at standard doses.

As knowledge of genetic variations in other enzymes and transporters involved in drug disposition increases, there is a need to develop strategies to evaluate the potential importance of these variations in drug development and clinical use. The guidance document from the European Medicines Agency (http://www.ema.europa.eu/pdfs/ human/pharmacogenetics/3764609endraft. pdf) aims to clarify requirements related to the application of pharmacogenetics in the study of the pharmacokinetics of investigational drugs, including issues such as the situations in which pharmacogenetic studies should be performed and the design of such studies. The deadline for comments is 31 October 2010. 